\title{
Mechanism of carrier generation in poly(phenylene vinylene): Transient photoconductivity and photoluminescence at high electric fields
}

\author{
D. Moses, H. Okumoto,* C. H. Lee, and A. J. Heeger \\ Institute for Polymers and Organic Solids, University of California, Santa Barbara, Santa Barbara, California 93106 \\ T. Ohnishi and T. Noguchi \\ Tsukuba Research Laboratory, Sumitomo Chemical Company, Ltd., 6 Kitahara, Tsukuba, Ibaraki, 300-32 Japan
}

(Received 19 May 1995; revised manuscript received 18 March 1996)

\begin{abstract}
The carrier generation mechanism in poly(phenylene vinylene), is addressed by studying the transient photoconductivity and the photoluminescence as a function of the external electric field $E$ in samples oriented by tensile drawing. The transient photocurrent is proportional to $E$ at low fields, but increases nonlinearly for $E>10^{5} \mathrm{~V} / \mathrm{cm}$. The field at which the photoconductivity becomes nonlinear (the onset field, $E_{0}^{\mathrm{pc}}$ ) depends on the degree of alignment: the higher the draw ratio, the lower $E_{0}^{\mathrm{pc}}$. The dependence of the photocurrent on $E$ is similar to the dependence of the dark current on $E$; both imply a field-dependent mobility (rather than fielddependent carrier generation). The onset field for the nonlinear photoconductivity is, however, different from the onset field for quenching the luminescence $\left(E_{0}^{\mathrm{pl}}\right)$. Thus, contrary to expectations for strongly bound neutral excitons as the elementary excitations, the high-field increase in photocurrent and the corresponding decrease in photoluminescence are not proportional, indicating that field-induced carrier generation is not significant. [S0163-1829(96)08231-8]
\end{abstract}

\section{INTRODUCTION}

The fundamental question concerning carrier photogeneration in poly(phenylene vinylene), PVV, can be succinctly stated: Are photocarriers created directly via interband photoexcitation or as a result of exciton dissociation? We address this issue by measuring the transient photocurrent $I_{p}$ (temporal resolution of $50 \mathrm{ps}$ ) and photoluminescence in the high-quality PPV samples oriented by tensile drawing, as a function of field $\left(E \leqslant 8 \times 10^{5} \mathrm{~V} / \mathrm{cm}\right)$ and temperature.

Although considerable effort has been devoted to attempts to unravel the three generic aspects of transient photoconductivity in conducting polymers (carrier photogeneration, carrier mobility, and carrier recombination), ${ }^{1}$ the conclusions remain controversial. It has been widely assumed that in low mobility materials (e.g., conducting polymers, amorphous semiconductors, and molecular crystals), there is a high probability that the geminate electron-hole pair will remain bound (an exciton) during the entire thermalization process. Therefore, models of carrier photogeneration for this class of materials, ${ }^{2-5}$ in particular, the Onsager model, ${ }^{6}$ have emphasized the importance of the Coulomb interaction in binding the pair and the role of the external field in processes that dissociate the pair into "free" mobile carriers. ${ }^{2-6}$

Extensive studies of fast transient photoconductivity of conducting polymers, in the subnanosecond time regime, have indicated, however, that different phenomena underlie the carrier generation. ${ }^{1,7-9}$ The experimental facts of particular importance are the following: (i) The fast transient photocurrent is independent of temperature ( $T$ ); (ii) the fast transient photocurrent is linearly proportional to the external field $(E)$; (iii) the fast transient photocurrent is linearly proportional to the light intensity; and (iv) the displacement current contribution is far too small to account for the photocurrent. ${ }^{8}$ (i) and (ii) imply that the quantum efficiency of carrier generation, $\eta$, is independent of $T$ and $E$; (iii) implies that the carrier generation is independent of the level of excitation. Thus, carriers are generated by a first-order process that cannot be attributed to interactions between excitations.

The photoconductivity data are consistent with photoexcitation of charged (positive and negative) polarons. Illumination by light with photon energy greater than the absorption edge generates carriers that promptly contribute to the photoconductivity, consistent with (i) through (iv) listed above, and with the sharp rise time of the transient photocurrent. As the carriers thermalize to the band edges, they may form weakly bound excitons (polaron pairs bound by the Coulomb interaction). This scenario is similar to that accepted for conventional semiconductors; in fact, (i) through (iv) are generally characteristic of photoconductivity in semiconductors where the electronic wave functions are delocalized and the electronic structure is described by band theory.

Although disorder leads to localization of the wave functions in semiconducting polymers, experiments have shown that in highly oriented and structurally ordered materials in which the macromolecules are chain extended and chain aligned, the excited state wave functions are delocalized over a minimum of fifty repeat units $(400 \AA) .{ }^{10}$ It is precisely because the excited state wave functions are delocalized over many structural repeat units that the semiconductor model is the proper starting point for a description of the excited states. For less well-ordered material, the mean localization length will be correspondingly smaller. One consequence of the disorder in conducting polymers is that the prompt mobility is temperature independent, rather than increasing with decreasing temperature, as is the case in crystalline semiconductors.

The agreement between the energy for onset of photoconductivity and the energy for onset of optical absorption implies that the exciton binding energy $E_{b}$ is rather small 
$\left(E_{b}<0.1 \mathrm{eV}\right){ }^{7-9}$ This method of determining $E_{b}$ has been clearly established, for example, from experimental studies of the polydiacetylenes. ${ }^{11}$ Moreover, Eckhardt et al. ${ }^{12}$ found that the electrochemically derived band gaps for PPV, poly(thienylene vinylene) and their alkoxy-substituted derivatives agree well with the band gaps obtained from optical absorption, indicating that in all cases $E_{b}$ is within the measurement error $\left(E_{b}<0.1 \mathrm{eV}\right)$. More recently, studies of the onset of emission, charge injection, and absorption in light-emitting electrochemical cells have shown that the binding energy is less than $0.1 \mathrm{eV} .{ }^{13}$

Nevertheless, the nature of electronic photoexcitations in PPV and its derivatives has remained controversial. ${ }^{14-16}$ Kersting et al. ${ }^{16}$ reported field-induced quenching of the transient photoluminescence in poly(phenyl-phenylene vinylene), PPPV. They found that the magnitude of the spectrally integrated luminescence decreases by about $30 \%$ in a field of $2 \times 10^{6} \mathrm{~V} / \mathrm{cm}$. They interpreted the field-induced quenching in terms of dissociation of excitons with a relatively large binding energy and concluded that the photocarriers are formed indirectly by exciton dissociation. ${ }^{16}$

With the goal of resolving this controversy, we have measured the transient photocurrent $I_{p}$ (temporal resolution of 50 ps) and photoluminescence in high-quality samples of PPV as a function of field $\left(E \leqslant 8 \times 10^{5} \mathrm{~V} / \mathrm{cm}\right)$ and temperature; high-field measurements of the dark current $I_{d}$ are included as well. We find a nonlinear dependence of the peak photocurrent $I_{p}$ on $E$ that appears at $E>5 \times 10^{4} \mathrm{~V} / \mathrm{cm}$ for oriented samples with a draw ratio of 10:1; higher fields are required for smaller draw ratios. Since the onset of nonlinearity depends on the degree of alignment, the nonlinearity implies an increase in carrier transport rather than an increase in carrier generation. High-field quenching of the photoluminescence is also observed. In order to investigate the correlation between the nonlinear photocurrent and the luminescence quenching, we have carefully measured the field dependence of the transient photocurrent and the field dependence of the luminescence in the same sample. Contrary to the predictions for strongly bound neutral excitons as the elementary excitations, the high-field increase in photocurrent and the corresponding decrease in photoluminescence are not proportional; in particular, the onset field for the nonlinear photoconductivity is significantly different from that for the luminescence quenching. These observations, in conjunction with the dependence of the dark current on $E$, imply that the high-field nonlinear increase in photoconductivity results from a field dependence of the mobility on $E$, consistent with recent hole mobility measurements in PPV. ${ }^{17}$

\section{EXPERIMENTAL METHODS}

The transient photoconductivity was measured using the Auston microstrip-switch technique. ${ }^{18}$ Gold microstrips were deposited on top of the PPV film leaving a gap of 10-20 $\mu \mathrm{m}$ between $600-\mu \mathrm{m}$-wide microstrips; a gold ground plane was deposited onto the back surface of an alumina substrate to form a transmission line with $50-\Omega$ impedance. One microstrip is biased with a dc voltage, and the other connected to the EG\&G PAR 4400 boxcar system fitted with a Tektronix S-4 sampling head. The PPV samples were freestanding films oriented by tensile drawing (draw ratio $l / l_{0}=1,2,3$, and 10), with thickness between 5 and 15 $\mu \mathrm{m}$. The films were placed on the alumina substrate so that the orientation axis (the draw axis) is parallel to the electric field within the gap between the two microstrips. A dye laser system (PRA LN105A) pumped with a PRA LN1000 $\mathrm{N}_{2}$ laser was used to produce 25-ps pulses at a photon energy of $\hbar \omega=2.92 \mathrm{eV}$ with a repetition rate of $5 \mathrm{~Hz}$. The overall temporal resolution of the measuring system is $\approx 50 \mathrm{ps}$. In some experiments, the laser light was polarized perpendicular to the orientation direction of the polymer film (and perpendicular to the direction of current flow) and in others the light was unpolarized.

Dark current measurements (versus $T$ and $E$ ) were carried out using a Keithley 487 picoammeter/voltage source.

In experiments involving high electric field, one must carefully consider the possibility of transient Joule heating due to the relatively large transient photocurrent that is generated. Our experiments were carried out under constant current conditions (at approximately $10^{-4} \mathrm{~A}$ ); i.e., as the electric field was increased, the light intensity was decreased so as to keep the photocurrent constant. By working at constant photocurrent, the maximum energy per pulse was limited to $Q<4 \times 10^{-10} \mathrm{~J}$. At a maximum of $Q<4 \times 10^{-10} \mathrm{~J}$, we estimate that Joule heating is not significant.

The field-induced luminescence quenching was measured on a sample for which the transient photoconductivity was measured as well, using a modulated argon laser as the light source and a lock-in amplifier to detect the emission; the photoluminescence signal was detected with a photomultiplier as photodetector. In order to minimize the effect of drift in the laser power and light detection system in the measurement of the luminescence quenching, the luminescence at zero field was measured after each measurement of the luminescence at high field.

\section{RESULTS AND DISCUSSION}

\section{A. Field dependence of the transient photoconductivity and dark current}

Figure 1 shows a typical transient photocurrent wave form, measured at room temperature in PPV with a draw ratio of $10\left(l / l_{0}=10\right)$ in response to a light pulse with intensity of about $0.1 \mu \mathrm{J} / \mathrm{cm}^{2}$, at $E=2.4 \times 10^{5} \mathrm{~V} / \mathrm{cm}$. The rise time indicates the temporal resolution of the measuring system.

Figure 2 shows the dependence of the peak transient photocurrent $\left(I_{p}\right)$ and that of the dark current $\left(I_{d}\right)$ on external field; both sets of data were obtained from the same sample at various temperatures. As noted in Sec. II, the light intensity was decreased as the field was increased so that the peak photocurrent remained roughly constant, at approximately $10^{-4} \mathrm{~A}$. Since the photocurrent is known to be linear in light intensity at all applied fields used in our experiments, the data presented in Fig. 2 are normalized to a constant light intensity. The nonlinear dependence of $I_{p}$ on $E$ was checked over a limited field range at high fields using a constant low-level light intensity to pump $I_{p}$ (this cannot be extended to low fields because the signal becomes too weak to detect).

The peak transient photocurrent is six orders of magnitude greater than the dark current. Moreover, while $I_{p}$ (top curve) is independent of temperature, $I_{d}$ (lower curves) rapidly de- 


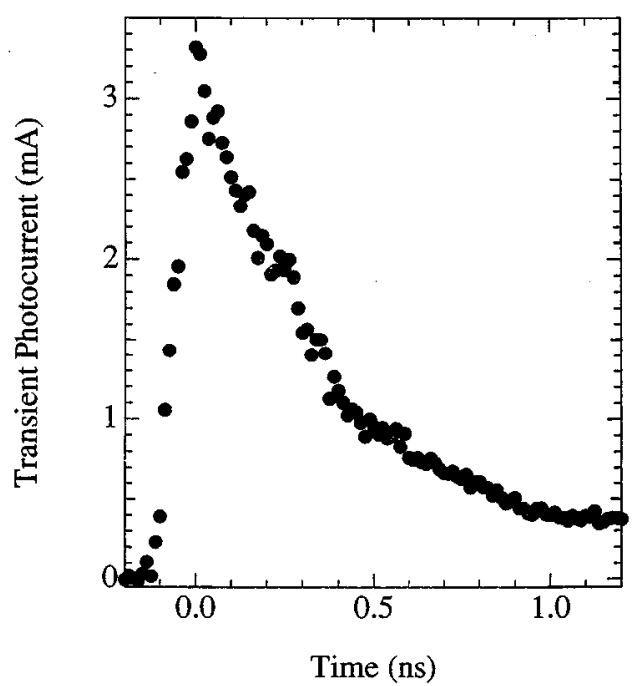

FIG. 1. Time-resolved transient photocurrent of oriented PPV; $\hbar \omega=2.92 \mathrm{eV} \quad\left(\approx 0.1 \mu \mathrm{J} / \mathrm{cm}^{2}\right.$ per 25 -ps pulse $), \quad T=300 \mathrm{~K}$, $E=2.4 \times 10^{5} \mathrm{~V} / \mathrm{cm}$.

creases as the temperature is lowered. The rate of decay of the photocurrent is independent of external field. The decay is weakly dependent on temperature; at low temperatures the long-lived tail slightly decreases while $I_{p}$ remains constant.

As shown in Fig. 2, the photocurrent and the dark current are linearly dependent on the field for $E<E_{0}^{\mathrm{pc}}=5 \times 10^{4} \mathrm{~V} / \mathrm{cm}$ $\left(l / l_{0}=10\right)$. The data are presented on a semilogarithmic plot in Fig. 3; for $E>E_{0}^{\mathrm{pc}}=5 \times 10^{4} \mathrm{~V} / \mathrm{cm}$, both $I_{p}$ and $I_{d}$ increase as $\exp (\alpha E)$. Comparing the slopes of the curves in Fig. 3, one finds that $\alpha_{p}<\alpha_{d}$, and that $\alpha_{d}$ increases slightly at low temperatures.

Although a similar dependence of the transient peak photocurrent and dark current on $E$ are observed for different

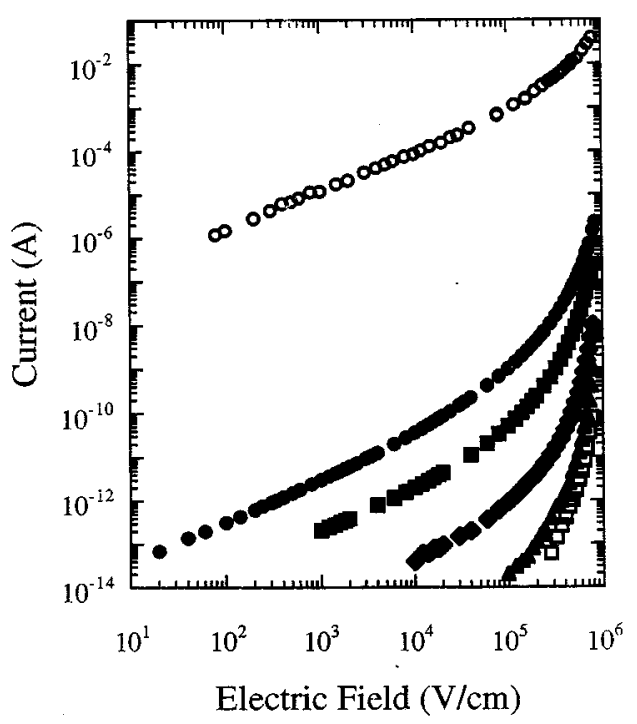

FIG. 2. The dependence of the peak transient photocurrent $\left(I_{p}\right)$ and dark current $\left(I_{d}\right)$ on external field at various temperatures in tensile drawn, oriented PPV, $l / l_{0}=10$. The top curve $(O)$ shows that $I_{p}$ is temperature independent. The lower curves represent $I_{d}: 300 \mathrm{~K}(\boldsymbol{\bullet}), 250 \mathrm{~K}(\boldsymbol{\square}), 200 \mathrm{~K}(\diamond), 150 \mathrm{~K}(\boldsymbol{\Delta}), 100 \mathrm{~K}$ $(\square)$.

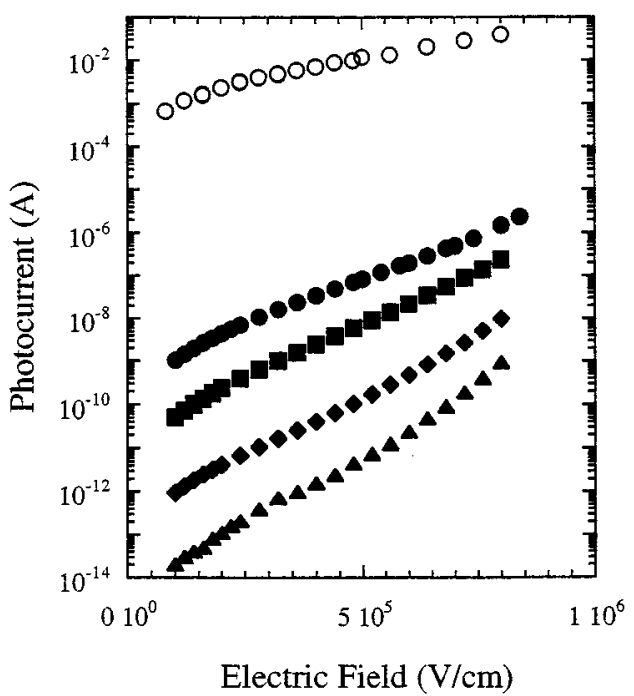

FIG. 3. The data from Fig. 2 in the high-electric-field regime on a semilogarithmic plot; the top curve $(O)$ represents the temperature-independent $I_{p}$, whereas the lower curves represent $I_{d}: 300 \mathrm{~K}(\bullet), 250 \mathrm{~K}(\boldsymbol{\square}), 200 \mathrm{~K}(\diamond), 150 \mathrm{~K}(\boldsymbol{\Delta})$.

draw ratios, the onset of photocurrent nonlinearity appears at somewhat higher fields; the field defining the onset of nonlinearity is $E_{0}^{\mathrm{pc}} \approx 10^{5} \mathrm{~V} / \mathrm{cm}$ in samples drawn to $l / l_{0}=3$ and $E_{0}^{\mathrm{pc}} \approx 1.7 \times 10^{5} \mathrm{~V} / \mathrm{cm}$ in polymer drawn to $l / l_{0}=2$. For nonoriented samples, the transient photocurrent remains linear at least to $E_{0}^{\mathrm{pc}}=5 \times 10^{5} \mathrm{~V} / \mathrm{cm}$ (the range of fields used for transient measurements on nonoriented PPV was limited to $E<5 \times 10^{5} \mathrm{~V} / \mathrm{cm}$ because of the larger dark current observed in this thicker, undrawn sample).

The dependence of the nonlinearity on sample orientation and structural order implies that the nonlinearity must arise from nonlinear carrier transport rather than nonlinear carrier generation. The current density $j_{p}$ is given by $j_{p}=n(t) e v_{d}=n(t) e \mu E$, where $n(t)$ is the number of carriers with charge $e, v_{d}$ is the drift velocity, and $\mu$ is the carrier mobility. The linear increase of $I_{p}$ with $E$ in oriented PPV at fields below $5 \times 10^{4} \mathrm{~V} / \mathrm{cm}$ is consistent with direct photogeneration of charge carriers that then move with a constant (field independent) mobility; in this linear regime, the carrier mobility is constant, and the quantum efficiency of carrier generation is independent of $E$. One might assume that the drift velocity is independent of $E$ and, therefore, that the quantum efficiency of carrier generation is proportional to $E$; however, the monotonic increase of the dark current with field is not consistent with such an assumption. Similar general behavior in a ladder-type $\pi$-conjugated polymer has been reported by Antoniadis et al. ${ }^{19}$

Field-dependent (increasing) mobilities have been observed at fields greater than $10^{5} \mathrm{~V} / \mathrm{cm}$ in organic field-effect transistors. ${ }^{20}$ Moreover, independent measurements of the mobility obtained from carrier transit time measurements in light-emitting diodes made of (nonoriented) PPV indicate a field-induced increase of the mobility for $E>6.7 \times 10^{4}$ $\mathrm{V} / \mathrm{cm} .{ }^{17}$ Thus, a corresponding increase of the carrier mobility is a plausible explanation of the nonlinear photoconductivity in oriented PPV. Based upon this assumption, the data in Fig. 3 indicate an exponential increase in mobility at high fields. ${ }^{21}$ Thus, the dependence of the photocurrent over the 
entire field regime is dominated by the behavior of the mobility; any increase in the carrier density in the high-field regime is relatively minor compared to the increase in the mobility.

The dependence of the onset field for the photocurrent nonlinearity on the degree of tensile drawing indicates that the field-induced increase in the mobility is more readily achieved in high-quality polymers. We note in this context that the magnitude of the peak transient photoconductivity depends on the degree of polymer orientation; $I_{p}$ is smaller in nonoriented PPV than in oriented PPV $\left(l / l_{0}=10\right)$ by about a factor of 4 , consistent with higher mobility in oriented samples.

Other sources of photoconductivity that are nonlinear in $E$ must also be considered, such as field-induced carrier detrapping, and carrier release from traps via "impact ionization." The onset of field-induced detrapping would occur when the carrier gains sufficient energy from the external field to overcome the trap binding energy. Since the strong temperature dependence of the dark current arises from shallow trapping with trap energies of order $k_{B} T\left(\approx 10^{-2} \mathrm{eV}\right)$, field-induced detrapping of carriers from shallow traps could occur as well. On the other hand, field-induced detrapping of carriers in the picosecond regime is unlikely because of the temperature independence of the transient photocurrent. The initial photocurrent measured in the picosecond regime is representative of pretrapping transport.

\section{B. Field dependence of the photoluminescence}

If the elementary excitations are strongly bound excitons, one expects free carriers only when they originate from exciton dissociation. As noted above, photoconductivity linear in $E$ is observed at fields that are orders of magnitude below the onset of photoluminescence quenching. Fast dissociation of excitons by defects could in principle yield the carriers responsible for the low-field photoconductivity. If this were the case, however, one would expect the luminescence to be quenched, analogous to the sensitization of the photoconductivity by the addition of $\mathrm{C}_{60} \cdot{ }^{9,22}$

In order to further explore the possibility of carrier generation via field-induced exciton dissociation, we look for a correlation between the nonlinear contribution to the photocurrent and the quenching of the photoluminescence. Assuming that each bound exciton dissociated by the field leads to free carriers, ${ }^{23,24}$

$$
\Delta \sigma(E) / \sigma_{\mathrm{pc}}^{0}=-A \Delta I_{L}(E) I_{L}^{0},
$$

where $\sigma_{\mathrm{pc}}^{0}$ is the low-field photoconductivity, $\Delta \sigma(E)$ is the field-dependent change in photoconductivity, $I_{L}^{0}$ is the lowfield luminescence intensity, and $\Delta I_{L}(E)$ is the change in photoluminescence intensity at high fields. Note that

$$
\Delta \sigma(E) / \sigma_{\mathrm{pc}}^{0}=\left[I_{\mathrm{pc}}(E)-I_{\mathrm{pc}}^{0}\right] / I_{\mathrm{pc}}^{0}=\Delta I_{\mathrm{pc}} / I_{\mathrm{pc}}^{0},
$$

where $I_{\mathrm{pc}}^{0}$ is the linear photocurrent extrapolated from the field regime below $4 \times 10^{4} \mathrm{~V} / \mathrm{cm}$.

We have measured the transient photoconductivity, dark current, and steady-state field-induced luminescence quenching at $T=77 \mathrm{~K})$ on the same sample $\left(l / l_{0}=2\right)$. The Auston switch configuration was used, with a sample length (gap

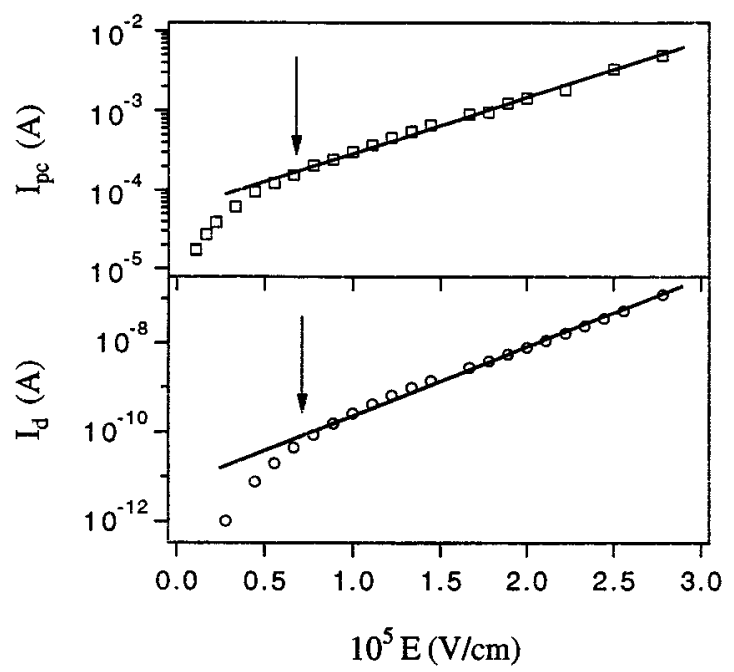

FIG. 4. The dependence of the transient photocurrent at $77 \mathrm{~K}$ on external field is compared to that of the dark current at the same temperature on a semilogarithmic graph. The arrows show the onset of the nonlinearity.

size) of approximately $18 \mu \mathrm{m}$. Since the excited-state lifetime in PPV is a few hundred picoseconds, and the transient photoconductivity spans a few hundred picoseconds (Fig. 1), we have measured the transient photoconductivity with 2-ns boxcar time gate. This procedure samples the early time photoresponse (i.e., at times particularly sensitive to the photogeneration process). Moreover, integrating over the 2-ns boxcar gate provides an accurate measurement of the response of the subnanosecond photocurrent with high signalto-noise ratio.

Figure 4 compares the field dependence of the transient photocurrent and the corresponding dark current for the sample with $l / l_{0}=2$. The data are plotted on a semilogarithmic graph in order to determine the onset field of the exponential component in $I_{\mathrm{pc}}$ and $I_{d}$. As the data indicate, within

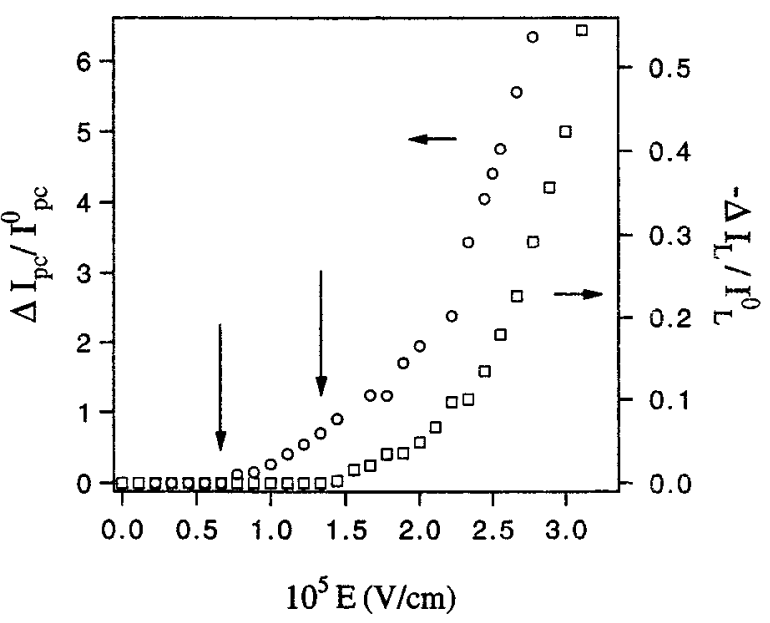

FIG. 5. The dependence of the normalized change in the transient photocurrent $\Delta I_{\mathrm{pc}} / I_{\mathrm{pc}}^{0}$ and the photoluminescence luminescence quenching $-\Delta I_{L}(E) / I_{L}^{0}$ on external field in oriented PPV $\left(l / l_{0}=2\right)$ at $77 \mathrm{~K}$. The arrows denote the onset of the nonlinearity in the photoconductivity (open circles) and the onset of the photoluminescence quenching (open squares). 


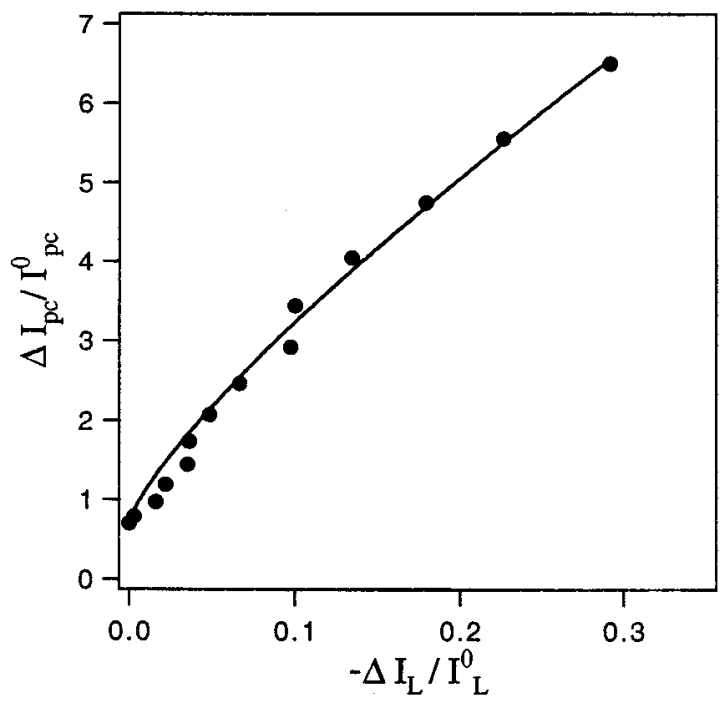

FIG. 6. The normalized change in the transient photocurrent $\left(\Delta I_{\mathrm{pc}} / I_{\mathrm{pc}}^{0}\right)$ is plotted vs the photoluminescence luminescence quenching $\left[-\Delta_{L}(E) / I_{L}^{0}\right]$. The solid curve is a fit to a power-law functional form of $y=A+B x^{\beta}$, where $y=\Delta I_{\mathrm{pc}} / I_{\mathrm{pc}}^{0}$, and $x=-\Delta I_{L}(E) / I_{L}^{0}$; the best fit to the power law yields $\beta=0.78$.

experimental uncertainty, the onset fields are identical, implying that the nonlinearity in the photocurrent and in the dark current have a common origin, i.e., the onset of the nonlinearity in the mobility, consistent with the sensitivity of the onset field to draw ratio and consistent with the fieldinduced mobility reported by Karg et al. ${ }^{17}$

The photoconductivity $\left(\Delta I_{\mathrm{pc}} / I_{\mathrm{pc}}^{0}\right)$ and the photoluminescence $\left[-\Delta I_{L}(E) / I_{L}^{0}\right]$ data obtained at $77 \mathrm{~K}$ are plotted versus the bias field in Fig. 5. The data indicate clearly that onset field for the nonlinear photocurrent, $E_{0}^{\mathrm{pc}}=0.77 \times 10^{5}$ $\mathrm{V} / \mathrm{cm}$, is lower by about $50 \%$ than the onset field of the luminescence quenching, $E_{0}^{\mathrm{pl}}=1.7 \times 10^{5} \mathrm{~V} / \mathrm{cm}$. Below $E_{0}^{\mathrm{pc}}$ the photocurrent is linearly dependent on $E$, and below $E_{0}^{\mathrm{pl}}$ the luminescence is field independent. At the highest electric fields employed in the transient photoconductivity experiment $\left(E=2.8 \times 10^{5} \mathrm{~V} / \mathrm{cm}\right),-\Delta I_{L}(E) / I_{L}^{0} \approx 0.30$, whereas the photocurrent increases beyond the linear extrapolation by a factor of $\approx 6.3$.

In Fig. $6, \Delta I_{\mathrm{pc}} / I_{\mathrm{pc}}^{0}$ is plotted versus $-\Delta I_{L}(E) I_{L}^{0}$. If carrier generation originates from exciton dissociation, a linear correlation should exist between $\Delta \sigma(E) / \sigma_{\mathrm{pc}}^{0}$ and $-\Delta I_{L}(E) I_{L}^{0}$. The solid curve in Fig. 6 corresponds to $y=A+B x^{\beta}$ where $y=\Delta I_{\mathrm{pc}} / I_{\mathrm{pc}}^{0}$ and $x=-\Delta I_{L}(E) / I_{L}^{0}$. The intercept $A$ arises from the different values for the onset field discussed above. The best fit to the power law yields $\beta=0.78$. Figures 5 and 6 demonstrate the absence of a linear correlation [Eq. (1)] between $\Delta I_{\mathrm{pc}} / I_{\mathrm{pc}}^{0}$ and $-\Delta I_{L}(E) / I_{L}^{0}$. The onset fields are different (the nonlinearity in the photoconductivity turns on at a lower field), and even above the onset, $\Delta I_{\mathrm{pc}} / I_{\mathrm{pc}}^{0}$ is sublinear with respect to $\sim \Delta I_{L}(E) / I_{L}^{0}$. This sublinear dependence is even more striking in the context of the field-induced increase in mobility discussed above. Given the increase in mobility, any residual change in the number of carriers $(\Delta n / n)$ as a function of $\sim \Delta I_{L}(E) / I_{L}^{0}$ is small.
To our knowledge, this is the first time an experiment has been carried out to test Eq. (1) using the subnanosecond transient photoconductivity; in experiments on other materials, the steady-state photoconductivity has been used to test Eq. (1). Since the steady-state photoconductivity is often dominated by processes that occur at times long after the photoluminescence decay time, using the transient photoconductivity is a more rigorous test of the correlation between carrier generation and luminescence quenching predicted for strongly bound excitons.

\section{Discussion on the mechanism of carrier generation and luminescence quenching}

The linear dependence of the transient photocurrent on $E$ in the low-field regime, at fields orders of magnitude below the onset of nonlinear transport (see Fig. 2), indicates a carrier generation mechanism independent of external field. This, in conjunction with (i) through (iv) listed in the Introduction, implies the following process for excitation in PPV: Illumination by light with photon energies greater than the absorption edge generates mobile carriers via interband excitation. While thermalizing toward the band edges to form self-localized charged polarons, these carriers promptly contribute to the transport, consistent with (i) through (iv), and with the sharp rise time of the transient photocurrent. This process is similar to that determined for conventional inorganic semiconductors; in fact, the above properties are standard features of photoconductivity in band semiconductors. As the carriers thermalize into states near the band edges, they form charged polarons that interact via the electronphonon interaction and the Coulomb interaction to form weakly bound polaron excitons.

Field-induced photoluminescence quenching is a general phenomenon, with different detailed mechanisms in different regimes. ${ }^{23,24}$ The absence of correlation between $\Delta \sigma(E) / \sigma_{\mathrm{pc}}^{0}$ and $-\Delta I_{L}(E) / I_{L}^{0}$ implies that field-induced dissociation of strongly bound excitons is not the mechanism responsible for the luminescence quenching.

The relatively low field required for the onset of luminescence quenching implies a weak exciton binding energy. Within the exciton model, luminescence quenching will occur when the charged carriers gain sufficient energy from the external field to overcome the exciton binding energy, $E_{b}$; i.e.,

$$
E_{b} \approx 2 a_{0} E_{0}^{\mathrm{pl}},
$$

where $2 a_{0}$ is the characteristic spatial size of the exciton wave function. Using $E_{0}^{\mathrm{pl}}=1.7 \times 10^{5} \mathrm{~V} / \mathrm{cm}$ and assuming that the exciton wave function in PPV extends over a few repeat units (for polydiacetylene, $2 a_{0} \approx 30 \AA$, see Ref. 25 and references therein), one obtains $E_{b} \approx 5 \times 10^{-2} \mathrm{eV}$, i.e., significantly smaller than that estimated previously $\left(E_{b} \approx 0.4-1\right.$ eV). ${ }^{16,26,27}$ Using $E_{0}^{\mathrm{pc}}=0.5 \times 10^{5} \mathrm{~V} / \mathrm{cm}$ as obtained from the most oriented samples, Eq. (4) yields $E_{b} \approx 2 \times 10^{-2} \mathrm{eV}$. Both values are of order $k_{B} T$ at room temperature.

In the limit of weak exciton binding energy, the "free carrier" excitations generated in semiconducting polymers 
are self-localized positive and negative polarons. Photoluminescence quenching would be expected when the polarons are separated by the applied field over a distance greater than the size of the polaron wave function; i.e., when

$$
\mu E \tau>L_{\text {polaron }}
$$

where $\mu$ is the transport mobility, $\tau$ is the time required for the onset of quenching, and $L_{\text {polaron }}$ is the spatial extent of the polaron wave function. Taking $L_{\text {polaron }} \approx 20 \AA, \tau \approx 50 \mathrm{ps}$, ${ }^{16}$ and $E \approx 2 \times 10^{5} \mathrm{~V} / \mathrm{cm}$, Eq. (4) yields $\mu>2 \times 10^{-2} \mathrm{~cm}^{2} / \mathrm{V} \mathrm{s}$. This value for the mobility would be considered high for steady-state conditions, but not unreasonable for times $<50$ ps after photogeneration, when pretrapping transport is dominant. Thus, field-induced quenching of the luminescence from mobile polaron pairs appears to be consistent with the experimental results.

Alternatively, many other processes are known to quench the luminescence. It is well known, for example, that injected carriers act as nonradiative recombination centers. ${ }^{28-30}$ The luminescence is quenched by doping. ${ }^{28}$ Dyreklev et al. ${ }^{29}$ showed that carriers injected into a polymer field-effect transistor act as nonradiative recombination centers. Quenching of the luminescence has been observed in PPV upon steadystate light illumination as well ${ }^{30}$ implying enhanced nonradiative decay due to photogenerated charge carriers. Trapped carriers would also be effective luminescence quenching centers; a relatively large density of trapped carriers is created especially when the sample temperature is comparable to the typical trap depth. Indeed, evidence for multiple trapping transport at long times in conducting polymers has been established by photoconductivity measurements. ${ }^{1}$

Deussen, Scheidler, and Bassler ${ }^{31}$ carried out measurements of the luminescence quenching in rectifying diodes (semiconducting polymer sandwiched between asymmetric electrodes). They observed that the luminescence quenching in forward bias is significantly larger than in reverse bias at the same field. This is particularly interesting since the higher luminescence quenching in forward bias is correlated with the higher photocurrent. Deussen, Scheidler, and Bassler $^{31}$ also found that the magnitude of luminescence quenching is reduced in polymer blends as the concentration of the active material (PPV) is decreased below about 10\%, eventually vanishing at $1 \%$. Although this concentration dependence would not be expected for field-induced luminescence quenching, it is consistent with carrier-induced quenching, which would go to zero at concentrations below the percolation threshold.

Thus, the luminescence quenching can be qualitatively understood to result from the high-field nonlinear transport, rather than vice versa. This conclusion is also consistent with the observation that the onset of luminescence quenching depends on the draw ratio of the oriented samples. Such a dependence is difficult to understand within the model expressed by Eq. (3), but follows naturally if nonlinear transport is the primary cause of the luminescence quenching.

\section{CONCLUSION}

In summary, the transient photocurrent in PPV is linear in $E$ for $E<E_{0}^{\mathrm{pc}}$ where $E_{0}^{\mathrm{pc}}$ depends on the draw ratio and the degree of orientation. For oriented samples with $l / l_{0}=10$, $E_{0}^{\mathrm{pc}}=5 \times 10^{4} \mathrm{~V} / \mathrm{cm}$. At fields greater than $E_{0}^{\mathrm{pc}}$, the transient and steady-state photoconductivity both increase exponentially with $E$. The better the polymer chain alignment, the lower the threshold field for the onset of nonlinear transport. The dependence of the nonlinearity on sample orientation and order, and the appearance of a similar exponential component in the dark current imply that the nonlinearity must arise from nonlinear carrier transport rather than nonlinear carrier generation; the nonlinear increase in transient photocurrent with field results from a field-induced increase in the transport mobility. Such a field-induced increase in mobility, observed in independent measurements, is expected to be sensitive to the quality of the material (i.e., chain alignment and density of defects) in agreement with our observations.

The transient photocurrent was measured over times (100-500 ps) comparable to the photoluminescence decay time. The linear dependence of the photocurrent on external field at fields below $5 \times 10^{4} \mathrm{~V} / \mathrm{cm}$ in PPV indicates the dominant importance of mobile charge carriers which are directly photogenerated rather than resulting from exciton dissociation. We find distinct and different fields for the onset of nonlinear photocurrent and luminescence quenching. Moreover, even at high fields where the photocurrent is nonlinear and luminescence quenching is observed, the two are not linearly correlated.

The observation of photocurrent response at low fields, the onset of photoconductivity at a photon energy that coincides with the absorption edge, and the absence of correlation between $\Delta \sigma(E) / \sigma_{\mathrm{pc}}^{0}$ and $-\Delta I_{L}(E) / I_{L}^{0}$ are all consistent with a model in which charged polarons (or polaron excitons with binding energy no greater than a few times $k_{B} T$ at room temperature), photogenerated through the interband $\pi-\pi^{*}$ transition are the primary photoexcitations in PPV.

\section{ACKNOWLEDGMENTS}

We thank Dr. U. Lemmer, Dr. D. Dick, and D. Vacar for useful discussions. This research was carried out with support from the Office of Naval Research under N0014-91-J1235 (Kenneth Wynne, Program Officer).
*Permanent address: National Institute of Materials and Chemical Research, Tsukuba, Ibaraki, Japan.

${ }^{1}$ D. Moses and A. J. Heeger, in Relaxation in Polymers, edited by T. Kobayashi (World Scientific, Singapore, 1993).

${ }^{2}$ M. Pope and C. E. Swenberg, Electronic Processes in Organic Crystals (Clarendon Press, Oxford, 1982).

${ }^{3}$ J. C. Knights and E. A. Davis, Phys. J. Chem. Solids 35, 543
(1974).

${ }^{4}$ D. M. Pai and R. C. Enck, Phys. Rev. B 11, 5163 (1975).

${ }^{5}$ R. C. Enck and G. Pfister, in Photoconductivity and Related Phenomena, edited by J. Mort and D. M. Pai (Elsevier, Amsterdam, 1976).

${ }^{6}$ L. Onsager, Phys. Rev. 54, 554 (1938). A recent theoretical extension of the Onsager solution has been developed by H. Scher 
and S. Rackovsky, J. Chem. Phys. 81, 1994 (1984).

${ }^{7}$ C. H. Lee, G. Yu, and A. J. Heeger, Phys. Rev. B 47, 15543 (1993).

${ }^{8}$ C. H. Lee, G. Yu, D. Moses, and A. J. Heeger, Phys. Rev. B 49, 2396 (1994).

${ }^{9}$ C. H. Lee, G. Yu, N. S. Sariciftci, A. J. Heeger, and C. Zhang, Synth. Met. 75, 127 (1995).

${ }^{10}$ T. W. Hagler, K. Pakbaz, and A. J. Heeger, Phys. Rev. B 49, 10968 (1994).

${ }^{11}$ R. R. Chance and R. H. Baughman, J. Chem. Phys. 64, 3889 (1976).

${ }^{12}$ H. Eckhart, L. W. Shacklette, K. Y. Jen, and R. L. Elsenbaumer, J. Chem. Phys. 91, 1303 (1989).

${ }^{13}$ Q. Pei, G. Yu, C. Zhang, Y. Yang, and A. J. Heeger, Science 269, 1086 (1995); G. Yu, Y. Yang, Y. Cao, Q. Pei, C. Zhang, and A. J. Heeger (unpublished).

${ }^{14}$ M. Gailberger and H. Bässler, Phys. Rev. B 44, 8643 (1991).

${ }^{15}$ E. L. Frankevich, A. A. Lymarev, I. Sokolik, F. E. Karasz, S. Blumstengel, R. H. Baughman, and H. H. Hörhold, Phys. Rev. B 46, 9320 (1992).

${ }^{16}$ R. Kersting, U. Lemmer, M. Deussen, H. J. Bakker, R. F. Mahrt, H. Kurz, V. I. Arkhipov, H. Bässler, and E. O. Göbel, Phys. Rev. Lett. 73, 1440 (1994).

${ }^{17}$ S. Karg, V. Dyakonov, M. Meier, W. Riess, and G. Paasch, Synth. Met. 67, 165 (1994).

${ }^{18}$ D. H. Auston, in Picosecond Optoelectric Devices, edited by C. H. Lee (Academic, New York, 1984), Chap. 4; J. Quantum Electron. 19, 636 (1983).

${ }^{19}$ H. Antoniadis, M. A. Abkowitz, J. A. Osaheni, S. A. Jenekhe, and M. Stolka, Synth. Met. 60, 149 (1993).
${ }^{20}$ A. Dodabalapur, L. Torsi, H. E. Katz, and A. J. Lovinger, Bull. Am. Phys. Soc. 40, 229 (1995); R. G. Kepler, J. M. Zeigler, L. A. Harrah, and S. R. Kurtz, Phys. Rev. B 35, 2818 (1987).

${ }^{21}$ S. Kar, Doctorate Dissertation, Bayreuth University, 1994. The mobility data in Ref. 17 are plotted as $\log \mu$ vs $E^{1 / 2}$. Over the limited field range, the data are equally well fitted to the exponential function $\mu \propto \exp \left(\alpha_{\mu} E\right)$.

${ }^{22}$ C. H. Lee, G. Yu, D. Moses, K. Pakbaz, C. Zhang, N. S. Sariciftci, A. J. Heeger, and F. Wudl, Phys. Rev. B 48, 15425 (1993).

${ }^{23}$ J. Ristein and G. Weiser, Philos. Mag. B 54, 533 (1986).

${ }^{24}$ R. Stachowitz, W. Fuhs, and K. Jahn, Philos. Mag. B 62, 5 (1990).

${ }^{25}$ B. I. Greene, J. Orenstein, R. R. Millard, and L. R. Williams, Phys. Rev. Lett. 58, 2750 (1987); L. Robbins, J. Orenstein, and R. S. Superfine, ibid. 56, 1850 (1986).

${ }^{26}$ U. Rauscher, H. Bassler, D. D. C. Bradley, and M. Hennecke, Phys. Rev. B 42, 9830 (1990).

${ }^{27}$ J. M. Leng, S. Jeglinski, X. Wei, R. E. Benner, Z. V. Vardeny, F. Guo, and S. Mazumdar, Phys. Rev. Lett. 72, 156 (1994); M. Chandross, S. Mazumdar, S. Jeglinski, X. Wei, Z. V. Vardeny, E. W. Kwock, and T. M. Miller, Phys. Rev. B 50, 14702 (1994).

${ }^{28}$ S. Hayashi, K. Kaneto, and K. Yoshino, Solid State Commun. 61, 249 (1987).

${ }^{29}$ P. Dyreklev, O. Inganäs, J. Paloheimo, and H. Stubb, J. Appl. Phys. 71, 2816 (1992).

${ }^{30}$ D. D. C. Bradley and R. H. Friend, J. Phys. Condens. Matter 1, 3671 (1989).

${ }^{31}$ M. Deussen, M. Scheidler, and H. Bassler (unpublished). 